\title{
Are we ready to adopt the European Association of Urology recommendations on multiparametric magnetic resonance imaging in the early detection of prostate cancer?
}

The evidence surrounding the role of multiparametric magnetic resonance imaging (mpMRI) in the early detection of prostate cancer has evolved to such an extent in recent years that it has led to an update of the European Association of Urology (EAU) recommendations regarding mpMRI [1]. These changes pertain to both biopsy-naïve men and those in whom prior negative biopsy was found. According to the new recommendations, there is now level la evidence to support the use of routine mpMRI prior to prostate biopsy in both of the aforementioned patient groups [1]. Further, if a Prostate Imaging and Reporting and Data System (PI-RADS) 3 or greater lesion is found, the recommendation for biopsy-naïve men is to perform targeted and systemic biopsy. However, for men with prior negative biopsy, only targeted biopsy is recommended. Although mpMRI has its limitations, undoubtedly, it now deserves a position in the early detection paradigm for suspected localised prostate cancer [2].

However, some significant questions emerge in light of these strong recommendations to use mpMRI as a triage tool upfront in men suspected of having early prostate cancer [3]. First, do we have enough confidence in the quality of mpMRI in each of our own institutions to follow the current recommendations? Second, do we have sufficient access to high-quality mpMRI in a timely fashion? Third, are resources in place to fund mpMRI at the scale required with such a common malignancy?

Australia and the UK have been early adopters of mpMRI. It is widely accepted as part of the armament in the early detection of prostate cancer and is reimbursed in this setting. Furthermore, with the recent publication of two landmark papers in The Lancet [4] and the New England Journal of Medicine [5] it comes as no surprise that mpMRI has reached the EAU guidelines. The question remains: are we as clinicians ready to adopt these recommendations, not just as 'early adopters', but as 'true believers'? Let us look at the evidence.

The first of these now well-known publications is the PROMIS study [4]. This was a prospective multicentre study of 576 men (mean age 63 years, mean prostate specific antigen [PSA] $7.1 \mathrm{ng} / \mathrm{mL}$ ) which set out to determine the diagnostic accuracy of mpMRI and transrectal ultrasoundguided (TRUS) biopsy of the prostate compared with a mapping template biopsy performed transperineally (TPMbiopsy) using a $5 \mathrm{~mm}$ sampling frame. All men underwent $1.5 \mathrm{~T}$ mpMRI (without endorectal coil) followed by TRUS biopsy and TPM-biopsy in that order. Clinically significant cancer was primarily defined as grade group 3 or greater (grade group 2 was incorporated in the secondary definition), or a maximum cancer core length of $6 \mathrm{~mm}$ or more. Of the 576 men, TPM-biopsy and TRUS biopsy detected clinically significant cancer in $230(40 \%)$ and 124 (22\%) men respectively. The authors reported the sensitivity and negative predictive value of mpMRI to detect grade group 2 cancer as $88 \%$ and $76 \%$. Seventeen clinically significant cancers seen on TPM-biopsy were missed on mpMRI, but all of these were grade group 2 or less. They concluded that an unnecessary biopsy might be avoided in $27 \%$ of patients if mpMRI was used to guide progression the decision to biopsy.

The second publication is the PRECISION study [5]. This was an international multicentre study of 500 men with a clinical suspicion of prostate cancer (mean age 64 years, median PSA $6.7 \mathrm{ng} / \mathrm{mL}$ ) who were randomised to standard 12-core TRUS biopsy pathway or an mpMRI pathway. Any man with an abnormal mpMRI (72\%) underwent targeted biopsy only with a median of four cores taken. Therefore, 
over a quarter of men avoided a biopsy based on a negative mpMRI. Clinically significant cancer was defined as the presence of a single core containing grade group 2 prostate cancer or above. The detection rate of clinically significant cancer in the TRUS arm and the targeted arm was $26 \%$ and $38 \%$ respectively. Detection of clinically insignificant cancer was $22 \%$ and $9 \%$ respectively. The authors concluded by advocating for the use of mpMRI in the diagnostic pathway of prostate cancer.

These studies certainly raise some important points for consideration. Firstly, in the PROMIS study there was no analysis performed to determine histopathological correlation with mpMRI region of interest because clinicians performing. TPM-biopsy were blinded to the mpMRI findings. However, other studies have previously reported on this. For example, a prospective randomised study comparing MRI/ultrasound fusion targeted biopsy with standard TRUS biopsy found that clinically significant cancer detection rates were higher in the targeted biopsy arm (43.9\% vs. $18.1 \%$ ) [6].

Secondly, mpMRIs were not reported using the PI-RADS scoring system but rather a Likert 1 to 5 scoring scale which has since been shown to be comparable to the PI-RADS. The consistency and skill of mpMRI reporting is of paramount importance in the use of mpMRI for early detection of prostate cancer. This represents one of the challenges in adopting the new EAU recommendations as a significant financial investment will be required to educate regional and lower volume mpMRI centres to achieve a consistently high standard of reporting. Individual centres will need to evaluate their own mpMRI and histopathological results to determine their predictive values. Involvement in a multidisciplinary meeting with urologist and pathologist is mandatory.

Thirdly, the cost analysis of mpMRI as a diagnostic tool to dictate biopsy is yet to be conducted. The savings from unnecessary biopsies and associated morbidity versus the cost of potentially missing a clinically significant cancer will need to be balanced.

Are we ready to adopt the EAU recommendations on mpMRI in the early detection of prostate cancer? We believe it is a step in the right direction towards minimising overdiagnosis of insignificant cancers while appropriately diagnosing significant cancers. However, as highlighted above, key infrastructure and training will be required to implement these recommendations.

\section{CONFLICTS OF INTEREST}

The authors have nothing to disclose.

Isaac A. Thangasamy ${ }^{12}$, Omar Alghazo, Declan G. Murphy ${ }^{1,3}$

Corresponding Author: Isaac A. Thangasamy ORCID: https:/orcid.org/0000-0003-1820-6114

${ }^{1}$ Division of Cancer Surgery, Peter MacCallum Cancer Centre, Melbourne,

${ }^{2}$ Faculty of Medicine, University of Queensland, Brisbane, ${ }^{3}$ Sir Peter MacCallum Department of Oncology, University of Melbourne, Parkville, Australia E-mail: ithangasamy@gmail.com

\section{REFERENCES}

1. Mottet N, Bellmunt J, Bolla M, Joniau S, Mason M, Matveev $\mathrm{V}$, et al. EAU guidelines: EAU-EANM-ESTRO-ESUR-SIOG guidelines on prostate cancer. Arnhem: EAU Guidelines Office; 2019.

2. Murphy DG, Padhani AR, Ost P. Adding colour to the grey zone of advanced prostate cancer. Eur Urol Focus 2019;5:123-4.

3. Nzenza T, Murphy DG. PRECISION delivers on the PROMIS of mpMRI in early detection. Nat Rev Urol 2018;15:529-30.

4. Ahmed HU, El-Shater Bosaily A, Brown LC, Gabe R, Kaplan $\mathrm{R}$, Parmar MK, et al. Diagnostic accuracy of multi-parametric MRI and TRUS biopsy in prostate cancer (PROMIS): a paired validating confirmatory study. Lancet 2017;389:815-22.

5. Kasivisvanathan V, Rannikko AS, Borghi M, Panebianco V, Mynderse LA, Vaarala MH, et al. MRI-targeted or standard biopsy for prostate-cancer diagnosis. N Engl J Med 2018;378:1767-77.

6. Porpiglia F, Manfredi M, Mele F, Cossu M, Bollito E, Veltri A, et al. Diagnostic pathway with multiparametric magnetic resonance imaging versus standard pathway: results from a randomized prospective study in biopsy-naïve patients with suspected prostate cancer. Eur Urol 2017;72:282-8. 